\title{
Cambios edáficos en islas de fertilidad y su importancia en el funcionamiento de un ecosistema del valle de Tehuacán Puebla, México
}

\author{
Edaphic changes in fertility islands and their importance for performance of an ecosystem in the \\ Tehuacan Valley, Puebla, Mexico
}

\author{
Daniel Jesús Muñoz Iniestra ${ }^{1 \neq}$, Mauricio Chávez Mosqueda ${ }^{1}$, \\ Héctor Octavio Godínez Álvarez ${ }^{1}$ y Norma Alejandra Cuéllar Arellano ${ }^{1}$
}

\footnotetext{
${ }^{1}$ Facultad de Estudios Superiores Iztacala, UNAM. Av. De los Barrios No. 1, Los Reyes Iztacala. 54090 Tlalnepantla, Estado de México, México. *Autor responsable (danimi@unam.mx)
}

\section{RESUMEN}

En los ecosistemas áridos y semiáridos la vegetación natural usualmente se presenta en forma de agrupaciones denominadas islas de fertilidad, separadas unas de otras por amplios espacios desprovistos de plantas. Se piensa que estas islas actúan como unidades funcionales básicas del ecosistema en su conjunto. Se ha observado que el suelo debajo de las islas de fertilidad muestra mejores condiciones de calidad y fertilidad en comparación con el suelo de afuera. El objetivo del trabajo fue evaluar la calidad del suelo dentro y fuera de las islas de fertilidad, así como contrastar propiedades físicas y químicas tanto del suelo interior como del exterior. Para esto se seleccionaron 25 islas de fertilidad de una terraza fluvial del valle de Zapotitlán, región semiárida de Tehuacán Puebla. En cada isla debajo del dosel del arbusto nodriza se tomaron tres muestras de suelo superficial, fuera de la isla alrededor se levantaron otras tres procediendo de la misma manera. Las muestras se analizaron en el laboratorio para evaluar nueve propiedades físicas y ocho químico-biológicas relacionadas con funciones ecológicas que el suelo realiza, estas propiedades se utilizaron para obtener un índice de calidad del suelo. Para el análisis de los datos se aplicó la prueba de t-test Student para muestras independientes. No hubo diferencias significativas en el índice de calidad entre los suelos fuera y dentro de las islas de fertilidad, sin embargo a nivel de propiedades individuales como: arena, densidad aparente, materia orgánica, $\mathrm{pH}$, magnesio, nitrógeno total y retención de humedad sí se presentaron diferencias significativas. Se concluye que la vegetación de la isla de fertilidad modifica

Cita recomendada:

Muñoz Iniestra, D. J., M. Chávez Mosqueda, H. O. Godínez Álvarez y N. A. Cuéllar Arellano. 2017. Cambios edáficos en islas de fertilidad y su importancia en el funcionamiento de un ecosistema del valle de Tehuacán Puebla, México. Terra Latinoamericana 35: 123-133. algunas propiedades y funciones del suelo para crear un microambiente más favorable, logrando que en su interior se reduzca el estrés hídrico y se propicie una realimentación constante de nutrientes, gracias a los mecanismos de captura y aporte de materia orgánica.

Palabras clave: calidad del suelo, zonas semiáridas, indicadores, Prosopis laevigata.

\section{SUMMARY}

In arid and semiarid ecosystems, natural vegetation commonly forms fertility islands, which are separated by wide areas lacking vegetation. It has been suggested that fertility islands are basic functional units in these ecosystems. Soil beneath fertility islands has higher quality and fertility than soil in areas lacking vegetation. The goals of this study were to evaluate soil quality and to compare soil physical and chemical properties inside and outside of fertility islands. We selected 25 fertility islands in an alluvial terrace in the Zapotitlan Valley, Tehuacan, Puebla. Three soil samples were collected beneath the nurse shrub of each island and in the areas lacking vegetation. Soil samples were analyzed in the laboratory to evaluate eight physical and nine chemicalbiological properties related to the ecological functions of soil. These properties were used to obtain a soil quality index. Data were analyzed with Student t-test for independent samples. Results showed that there were no significant differences in the soil quality index. However, some soil properties such as sand, apparent density, organic matter, $\mathrm{pH}$, magnesium, total nitrogen, and humidity differed between soils inside and outside 
fertility islands. We concluded that fertility islands modify some soil properties and functions creating more favorable microhabitats; they reduce water stress and provide nutrients as a result of the mechanisms for capturing and providing organic matter.

Index words: soil quality, semiarid zones, indicators, Prosopis laevigata.

\section{INTRODUCCIÓN}

En los ecosistemas áridos y semiáridos la vegetación natural usualmente se manifiesta como un mosaico que incluye un patrón de parches constituidos por agrupaciones vegetales con biomasa relativamente alta (islas de fertilidad o islas de recursos) dispersos en una matriz de suelo carente de vegetación o cubierto de costras microbióticas (Westoby et al., 1989; Turner y Brown, 1994; Peters et al., 2006). Este patrón de mosaico puede presentarse en paisajes relativamente homogéneos y permanecer así por largo tiempo, lo que sugiere que los diferentes tipos de parches representan estados alternativos estables (Holmgren y Scheffer, 2001; Herrick et. al., 2002). Según lo que proponen Westoby et al. (1989) la vegetación natural de las regiones secas cuando es alterada o modificada, puede transitar a diferentes estados alternos transitorios que pueden permanecer estables por largos periodos dependiendo de la intensidad y dirección de las fuerzas modificadoras. Por otra parte el suelo protegido debajo de parches densos de plantas, islas de fertilidad (IF), al parecer presenta mejores condiciones de calidad y fertilidad en comparación con el suelo fuera del dosel (Wezel et al., 2000; Tewksbury y Lloyd, 2001; Bestelmeyer et al., 2006, Bonanomi et al., 2007, Ridolfi et al., 2008).

Las IF son importantes porque regulan el funcionamiento de los ecosistemas en muchas zonas áridas y semiáridas, debido a que disminuyen el estrés hídrico y dentro de ellas se da la transformación de materia y energía regulando la cantidad de nitrógeno, carbono y fósforo que se almacena y mineraliza en el suelo, permitiendo con esto la productividad del ecosistema (Whitford, 2002; Bonanomi et al., 2007). Las IF comprenden uno o varios árboles o arbustos generalmente leguminosas y plantas establecidas bajo su dosel. Se piensa que actuan como unidades funcionales básicas que permiten la estabilidad y productividad del ecosistema en su conjunto y de paisajes completos en las regiones áridas y semiáridas (Tielborger y Kadmon, 2000; Whitford, 2002; Berg y Steinberger, 2012). La base funcional de las islas radica en una relación de nodricismo, donde plantas de diferentes especies están protegidas por un arbusto de otra especie, los arbustos crean microambientes más favorables para otros organismos (Flores y Jurado, 2003). Sin embargo, esta relación que por lo regular resulta ser positiva, con el trancurso del tiempo se puede convertir en negativa cuando la especie protegida interfiere o afecta el desarrollo de la planta nodriza (McAuliffe, 1988).

La presencia de una planta nodriza es escencial para que estas islas funcionen y la vegetación persista, la sombra bajo el dosel determina que las temperaturas del aire y del suelo sean más bajas reduciendo considerablemente la evapotranspiración lo que disminuye la pérdida de agua, por lo que las plántulas experimentan menos estrés hídrico y térmico (Titus et al., 2002; Schade y Hobbie, 2005). Por otra parte los arbustos actúan como trampa colectora de sedimentos y restos orgánicos transportados por el agua y viento. Estos materiales que se acumulan bajo la copa de los arbustos proveen de un mejor sustrato para las plantas protegidas que el que se encuentra en el suelo abierto. La distribución vertical y horizontal de nutrientes del suelo está estrechamente relacionada con la distribución de la vegetación (Tewksbury et al., 2001). Al interior de la isla las interacciones suelo-planta juegan un rol determinante para todos los habitantes de la isla. La hojarasca depositada bajo el dosel permite el enriquecimiento del suelo (Aber y Melillo, 2001).

La relación suelo planta al interior de las IF ha sido poco estudiada y entendida, se desconocen los mecanismos de influencia en el suelo de la IF, pero seguramente están relacionados con el acceso y disponibilidad de agua y nutrientes en las dierentes unidades geomorfológicas. El suelo fuera de la isla tiene un funcionamiento distinto presentando menor calidad y mayores condiciones de estrés lo que disminuye las posibilidades de establecimiento y crecimiento de plántulas y por lo tanto la cobertura vegetal, las IF sostienen plantas mucho más grandes y con más eficiencia de producción que los suelos pobres en nutrientes de las áreas abiertas (Schade y Hobbie, 2005; Bonanomi et al., 2007).

El conocimiento de las relaciones y procesos que se dan al interior de las IF es fundamental tanto para recuperar ecosistemas dañados por actividades humanas en las regiones secas, así como para 
implementar sistemas de manejo y conservación en estos ecosistemas, en este sentido, el objetivo del presente trabajo fue entender la relación suelo planta al interior de las islas de fertilidad de una región semiárida del Valle de Tehuacán Puebla, México. Para ello fue necesario obtener un índice de calidad del suelo del interior de las islas de fertilidad y compararlo con la calidad del suelo del exterior. Al mismo tiempo se buscó identificar que propiedades del suelo son las más determinantes en el funcionamiento de las IF.

\section{MATERIALES Y MÉTODOS}

\section{Área de Estudio}

El área en estudio fue un fragmento de terraza fluvial del río Zapotitlán en el Nor-noreste del valle de Tehuacán, en la municipalidad de Zapotitlán Salinas Puebla, México. La coordenada del punto medio del área es $18^{\circ} 19^{\prime} 18.38^{\prime \prime} \mathrm{N}$ y $97^{\circ} 27^{\prime} 27.09^{\prime \prime} \mathrm{O}$ con una elevación de $1460 \mathrm{~m}$, con una superficie aproximada de 2 ha. El clima del área según el sistema Köppen modificado por García (2004) es seco, semi-cálido (Bsohw(e) gw"), con una temperatura media promedio anual de $21^{\circ} \mathrm{C}$, la precipitación total anual promedio es de $446.8 \mathrm{~mm}$. Los suelos de las terrazas son Fluvisoles calcáricos, son suelos profundos con textura que fluctúa entre franca, franca arcillosa y franca limosa, no hay pedregosidad, la estructura es masiva y dura en la superficie con presencia de costras físicas delgadas y microbióticas (Muñoz et al., 2009). La cubierta vegetal de las terrazas está conformada por un matorral espinoso de mezquite (Prosopis laevigata) y palo verde (Parkinsonia praecox), que se distribuye de manera irregular formando un mosaico constituido por parches de vegetación de diferente tamaño (islas de fertilidad) separados por suelo desprovisto de plantas que se alternan con sitios fuertemente erosionados y parcelas agrícolas de temporal activas y en abandono (Figura. 1). Las terrazas fluviales han estado y están sujetas a una fuerte presión por parte de la gente de la localidad ya que son utilizadas para la agricultura, el pastoreo de ganado menor y obtención de leña, lo cual ha causado un fuerte deterioro a este sistema natural, que se manifiesta por la pérdida de cobertura vegetal, fuerte erosión del suelo y agotamiento de las tierras de cultivo (UBIPRO, 2007; Muñoz et al., 2009).

\section{Muestreo}

El muestreo del suelo se realizó en un fragmento de terraza fluvial la cual tiene un relieve casi llano donde la pendiente no sobre pasa a $1.5^{\circ}$, este sitio corresponde a un parche de matorral abierto muy deteriorado donde el único uso que hace la gente de la localidad es la extracción de leña, la cual se obtiene del corte de ramas viejas o muertas de los arbustos evitando con esto cortar todo el arbusto ya que quedan pocos individuos.

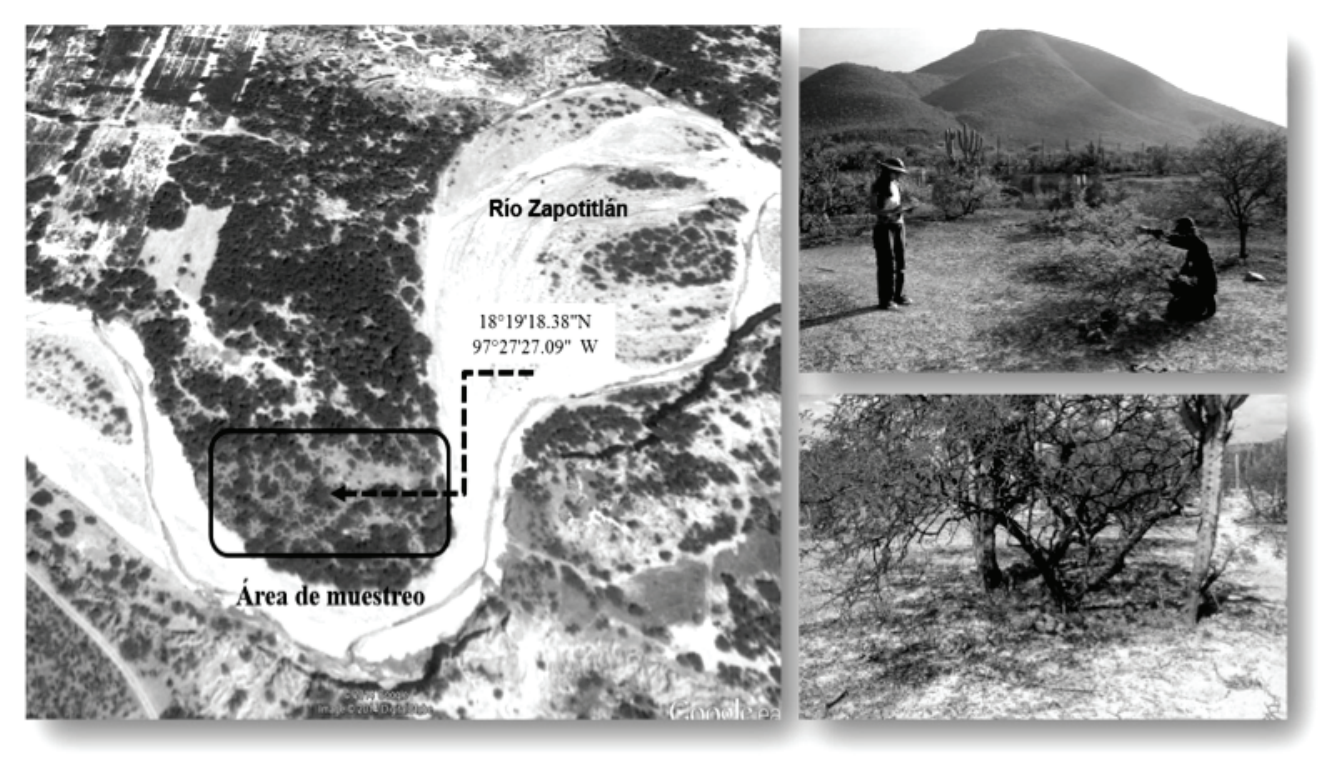

Figura 1. Terraza fluvial donde se realizó el estudio e imágenes de las islas de fertilida en Zapotitlán Salinas Puebla. 
Se seleccionaron y georreferenciaron 25 IF, en todas ellas el arbusto nodriza fue el mezquite (Prosopis laevigata), las islas fueron heterogéneas en cuanto a tamaño y altura de la nodriza. En el interior de cada una de ellas por debajo del dosel de la nodriza, se tomaron tres muestras de suelo superficial $(20 \mathrm{~cm})$ sin incluir la hojarasca, las cuales se homogenizaron y mezclaron para formar una muestra compuesta. De igual modo se tomó otra muestra compuesta pero fuera de cada isla en suelo abierto donde sólo prevalecía una cubierta de costras físicas y biológicas (suelo desnudo). Tanto fuera, como dentro del dosel del mezquite se tomó la temperatura edáfica y la atmosférica.

\section{Análisis Químicos y Físicos}

Las propiedades que se determinaron fueron: textura con el método del hidrómetro, densidad aparente por la técnica del cilindro metálico (USDA, 1999), densidad real por el método del picnómetro, conductividad eléctrica, materia orgánica, $\mathrm{pH}$ con potenciómetro digital, capacidad de intercambio catiónico total por el método volumétrico del versenato, calcio y magnesio intercambiables), sodio y potasio intercambiables por espectrofotometría de flama, nitrógeno total por el método semi-microKjeldahl, fósforo asimilable, humedad al momento, con sensor digital de campo (Aquater M300 digital) y temperatura (termómetro de suelos marca Taylor). Algunas variables fueron calculadas: capacidad de campo y punto de marchitez permanente (obtenidos con la ecuación de Bodman y Mahmud, citada por Silva et al., 1988), agua aprovechable por diferencia entre la capacidad de campo y el punto de marchitez permanente (Silva et al., 1988), relación agua-aire dividiendo la capacidad de campo entre la porosidad total (Skopp et al., 1990), capacidad de aireación o de drenaje restando el volumen de agua a capacidad de campo de la porosidad total (Silva et al., 1988; Skopp et al., 1990).

\section{Análisis de Datos}

Para la obtención del índice de calidad total del suelo (ICTS), se construyó un modelo que utiliza dos subíndices (Cuadro 1), esto con la finalidad de evaluar por separado las propiedades que tienen que ver con el funcionamiento físico-hídrico del suelo
(ÍCF) y las que se relacionan con las funciones químico biológicas del suelo. El índice y subíndices se alimentan con valores normalizados que van en una escala de 0 a 1 obtenidos de los valores reales (Andrews et al., 2002; Cantú et al., 2007). Para hacer la normalización las propiedades fueron clasificadas en tres categorías, en la primera que se denomina "mayor es mejor" se agruparon aquellas propiedades en donde lo más conveniente para tener una buena calidad del suelo es que estas presenten valores elevados, tal es el caso del nitrógeno ya que entre más se tenga de este nutriente mejor será el funcionamiento biológico del suelo y mayor beneficio habrá para la productividad. En el segundo grupo "menor es mejor" se colocaron propiedades en donde lo más conveniente para la calidad del suelo es que estas tengan un valor bajo, un ejemplo de esto puede ser la salinidad o la densidad aparente. En la tercera categoría se agruparon las denominadas independientes, en donde no es conveniente que tengan valores ni altos ni bajos, tal es el caso del $\mathrm{pH}$, la granulometría y otras. En el Cuadro 1 se presenta el listado completo de las propiedades utilizadas y su categorización correspondiente.

La normalización de las propiedades de la categoría "mayor es mejor" se realizó por medio de la ecuación (1) Vn= Im - Imin / Imax - Imin. Para la normalización de las propiedades denominadas "menor es mejor" se aplicó la ecuación (2) Vn = 1- (Imin - Imin / Imax-Imin), donde $\mathrm{Vn}=$ valor normalizado, $\mathrm{Im}=$ valor promedio del indicador, Imax = valor máximo del indicador, Imin = valor mínimo del indicador (Cantú et al., 2007). Las propiedades denominadas como independientes se normalizaron haciendo una consulta bibliográfica de expertos (USDA, 1999; Shepher, 2000; Andrews, 2002; Reynolds et al., 2002; Gugino et al., 2009) donde se revisaron diferentes criterios para evaluar estas propiedades, con esta información se construyeron cuadros de tabulación (Cuadro 2). Para calcular cada subíndice de cada muestra se sumaron todos los valores normalizados de las propiedades incluidas en ese subíndice y posteriormente se obtuvo el promedio. Para calcular el ICTS, se sumaron los valores de los dos subíndices y por último se promediaron (Cuadro 1). Al final se evaluó la calidad del suelo utilizando los criterios de Cantú (2007).

Para el análisis estadístico se utilizó el programa SP22 versión 21 con el que se obtuvo la media, desviación y error estándar, para la comparación del 
Cuadro 1. Ejemplo del procedimiento seguido para obtener los subíndices e índice de calidad total.

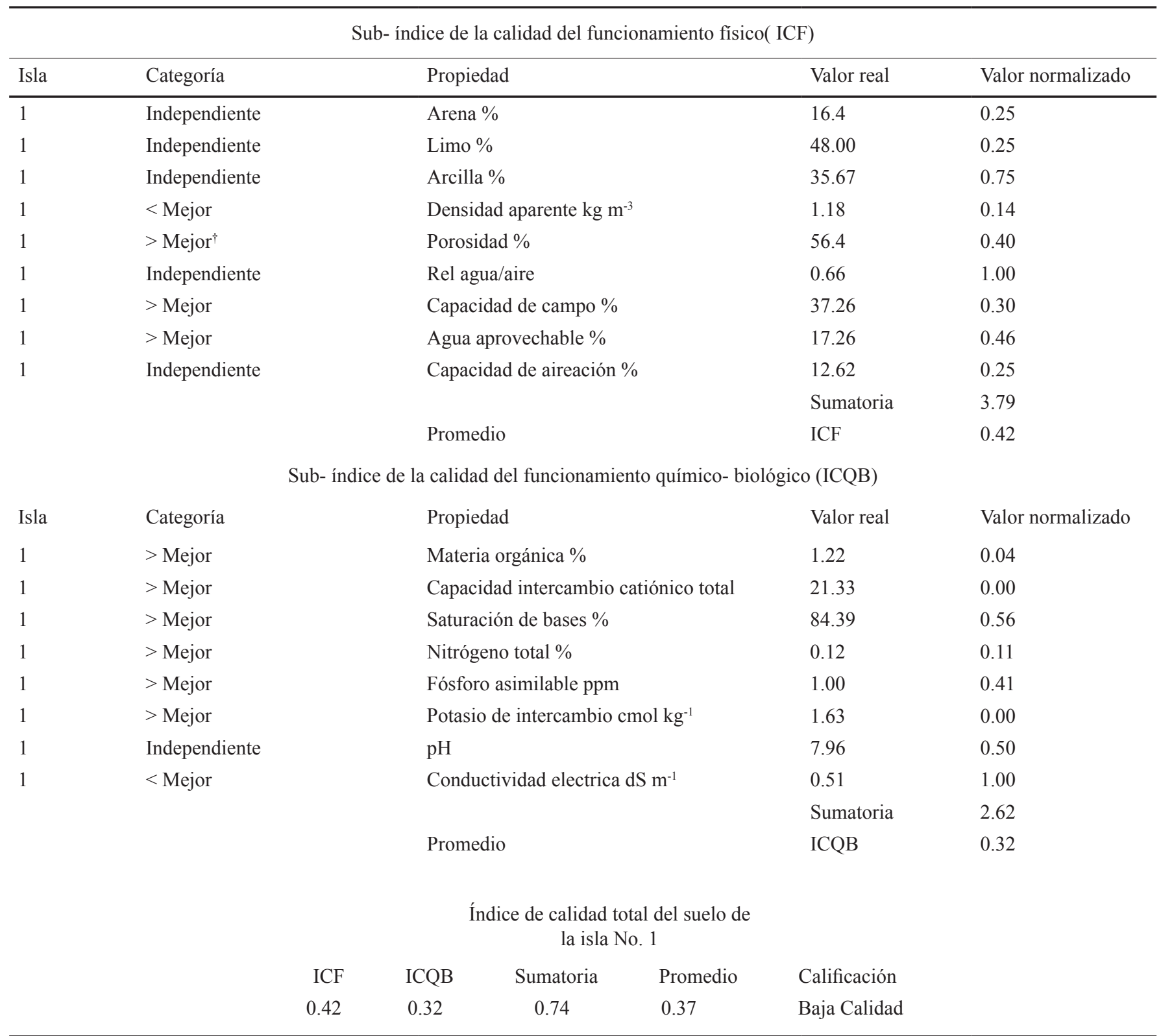

† En la categoría "mayor es mejor" se incluyeron propiedades en donde lo más conveniente para tener una buena calidad del suelo es que estas presenten valores elevados; por el contrario en la categoría "menor es mejor" se agruparon propiedades donde lo más conveniente para la calidad el suelo es que tengan valores bajos. La categoría independiente comprende propiedades en donde no es conveniente que tengan valores ni altos ni bajos por ejemplo el $\mathrm{pH}$.

índice de calidad del suelo y las propiedades evaluadas de las dos condiciones o factores, se utilizó una prueba de t-test Student para muestras independientes, antes de aplicar la prueba se verificó que los datos cumplían con el supuesto de normalidad, cuando no fue así se normalizaron transformándolos a una función de arcoseno (Zar, 1999). La evaluación de la calidad total del suelo se realizó según los criterios establecidos por Cantú (2007).

\section{RESULTADOS Y DISCUSIÓN}

\section{Calidad del Suelo}

El índice promedio de calidad total del suelo (ICTS) (Cuadro 3), no fue significativamente diferente entre dentro y fuera de las islas (gl.:48; sig: 0.471 para $\alpha<0.05$ ). De acuerdo con los criterios de valoración propuestos por Cantú (2007) ambas condiciones 
Cuadro 2. Criterios establecidos para la normalización de propiedades consideradas como "independientes".

\begin{tabular}{|c|c|c|c|c|c|c|c|c|}
\hline \multicolumn{5}{|c|}{ Criterios para la normalización de la textura } & \multicolumn{4}{|c|}{ Criterios para la normalización del pH } \\
\hline $\begin{array}{l}\text { Propiedad } \\
\%\end{array}$ & $\begin{array}{l}\text { Valor } \\
\text { mínimo }\end{array}$ & $\begin{array}{c}\text { Valor } \\
\text { máximo }\end{array}$ & $\begin{array}{c}\text { Valor } \\
\text { normalizado }\end{array}$ & Calificación & $\begin{array}{l}\text { Valor } \\
\text { mínimo }\end{array}$ & Valor máximo & $\begin{array}{c}\text { Valor } \\
\text { normalizado }\end{array}$ & Calificación \\
\hline Arena & 1 & 10 & 0.00 & MD & & $<5$ & 0.00 & MD \\
\hline Arena & 11 & 20 & 0.25 & $\mathrm{D}$ & 5 & 5.4 & 0.25 & $\mathrm{D}$ \\
\hline Arena & 21 & 30 & 0.50 & M & 5.5 & 5.9 & 0.50 & M \\
\hline Arena & 31 & 40 & 0.75 & $\mathrm{~F}$ & 6 & 6.4 & 0.75 & $\mathrm{~F}$ \\
\hline Arena & 41 & 50 & 1.00 & MF & 6.5 & 7.5 & 1.00 & MF \\
\hline Arena & 51 & 60 & 0.75 & $\mathrm{~F}$ & 7.6 & 7.9 & 0.75 & $\mathrm{~F}$ \\
\hline Arena & 61 & 70 & 0.50 & M & 8 & 8.5 & 0.50 & M \\
\hline Arena & 71 & 80 & 0.25 & $\mathrm{D}$ & 8.6 & 9.0 & 0.25 & $\mathrm{D}$ \\
\hline Arena & 81 & 100 & 0.00 & MD & $>9$ & & 0.00 & MD \\
\hline Limo & 1 & 5 & 0.00 & MD & & terios valoración ca & acidad de aire & ión \\
\hline Limo & 6 & 15 & 0.25 & $\mathrm{D}$ & $\begin{array}{c}\text { Valor } \\
\text { mínimo \% }\end{array}$ & Valor máximo \% & $\begin{array}{c}\text { Valor } \\
\text { normalizado }\end{array}$ & Calificación \\
\hline Limo & 16 & 20 & 0.75 & $\mathrm{~F}$ & $>56$ & & 0.00 & MD \\
\hline Limo & 21 & 30 & 1.00 & MF & 45 & 55 & 0.25 & $\mathrm{D}$ \\
\hline Limo & 31 & 40 & 0.50 & M & 39 & 44 & 0.50 & M \\
\hline Limo & 41 & 60 & 0.25 & $\mathrm{D}$ & 33 & 38 & 0.75 & $\mathrm{~F}$ \\
\hline \multirow[t]{2}{*}{ Limo } & $>60$ & & 0.00 & MD & 30 & 39 & 1.00 & MF \\
\hline & & & & & 21 & 26 & 0.75 & $\mathrm{~F}$ \\
\hline Arcilla & 1 & 10 & 0.25 & $\mathrm{D}$ & 21 & 20 & 0.50 & M \\
\hline Arcilla & 11 & 15 & 0.50 & M & 10 & 20 & 0.25 & $\mathrm{D}$ \\
\hline Arcilla & 16 & 20 & 0.75 & $\mathrm{~F}$ & & $<9$ & 0.00 & MD \\
\hline Arcilla & 21 & 30 & 1.00 & MF & & & & \\
\hline Arcilla & 31 & 35 & 0.75 & $\mathrm{~F}$ & \multicolumn{4}{|c|}{ Criterios valoración capacidad agua/aire } \\
\hline Arcilla & 36 & 40 & 0.50 & M & $\begin{array}{c}\text { Valor } \\
\text { mínimo \% }\end{array}$ & Valor máximo \% & $\begin{array}{c}\text { Valor } \\
\text { normalizado }\end{array}$ & Calificación \\
\hline Arcilla & 41 & 60 & 0.25 & $\mathrm{D}$ & $<0.45$ & & 0.00 & M \\
\hline \multirow[t]{8}{*}{ Arcilla } & 61 & 100 & 0.00 & MD & 0.45 & 0.49 & 0.25 & $\mathrm{D}$ \\
\hline & & & & & 0.5 & 0.55 & 0.50 & M \\
\hline & & & & & 0.56 & 0.60 & 0.75 & $\mathrm{~F}$ \\
\hline & & & & & 0.61 & 0.70 & 1.00 & MF \\
\hline & & & & & 0.71 & 0.75 & 0.75 & $\mathrm{~F}$ \\
\hline & & & & & 0.76 & 0.80 & 0.50 & M \\
\hline & & & & & 0.81 & 0.86 & 0.25 & $\mathrm{D}$ \\
\hline & & & & & & $>0.86$ & 0.00 & MD \\
\hline
\end{tabular}


Cuadro 3. Subíndices de calidad física (ICF), químico biológica (ICQB) e índice de calidad total del suelo (ICTS), dentro y fuera de las islas de fertilidad (IF).

\begin{tabular}{|c|c|c|c|c|c|c|}
\hline \multirow{2}{*}{ Isla } & \multicolumn{3}{|c|}{ Dentro } & \multicolumn{3}{|c|}{ Fuera } \\
\hline & ICF & ICQB & ICTotal & ICF & ICQB & ICTotal \\
\hline 1 & 0.422 & 0.338 & 0.373 & 0.595 & 0.427 & 0.511 \\
\hline 2 & 0.555 & 0.610 & 0.583 & 0.483 & 0.461 & 0.472 \\
\hline 3 & 0.635 & 0.610 & 0.623 & 0.398 & 0.535 & 0.467 \\
\hline 4 & 0.609 & 0.352 & 0.480 & 0.457 & 0.484 & 0.471 \\
\hline 5 & 0.409 & 0.393 & 0.401 & 0.464 & 0.388 & 0.426 \\
\hline 6 & 0.464 & 0.397 & 0.431 & 0.471 & 0.649 & 0.560 \\
\hline 7 & 0.660 & 0.566 & 0.613 & 0.567 & 0.552 & 0.559 \\
\hline 8 & 0.520 & 0.546 & 0.533 & 0.507 & 0.571 & 0.539 \\
\hline 9 & 0.617 & 0.476 & 0.546 & 0.589 & 0.589 & 0.589 \\
\hline 10 & 0.444 & 0.422 & 0.433 & 0.512 & 0.604 & 0.558 \\
\hline 11 & 0.497 & 0.462 & 0.480 & 0.463 & 0.516 & 0.489 \\
\hline 12 & 0.589 & 0.501 & 0.545 & 0.530 & 0.569 & 0.549 \\
\hline 13 & 0.554 & 0.491 & 0.522 & 0.411 & 0.534 & 0.472 \\
\hline 14 & 0.497 & 0.378 & 0.437 & 0.541 & 0.819 & 0.680 \\
\hline 15 & 0.512 & 0.534 & 0.523 & 0.534 & 0.580 & 0.557 \\
\hline 16 & 0.445 & 0.885 & 0.665 & 0.594 & 0.491 & 0.543 \\
\hline 17 & 0.412 & 0.531 & 0.471 & 0.463 & 0.542 & 0.503 \\
\hline 18 & 0.491 & 0.423 & 0.457 & 0.515 & 0.561 & 0.538 \\
\hline 19 & 0.622 & 0.556 & 0.589 & 0.573 & 0.562 & 0.567 \\
\hline 20 & 0.646 & 0.502 & 0.574 & 0.545 & 0.684 & 0.615 \\
\hline 21 & 0.417 & 0.510 & 0.463 & 0.504 & 0.599 & 0.551 \\
\hline 22 & 0.606 & 0.634 & 0.620 & 0.555 & 0.529 & 0.542 \\
\hline 23 & 0.606 & 0.673 & 0.640 & 0.452 & 0.574 & 0.513 \\
\hline 24 & 0.603 & 0.519 & 0.561 & 0.720 & 0.636 & 0.678 \\
\hline 25 & 0.569 & 0.596 & 0.583 & 0.527 & 0.665 & 0.596 \\
\hline Promedio & 0.538 & 0.517 & 0.528 & 0.518 & 0.564 & 0.542 \\
\hline Error Std & 0.016 & 0.023 & 0.015 & 0.014 & 0.017 & 0.012 \\
\hline
\end{tabular}

evaluadas presentan un suelo con moderada calidad. Al observar y comparar los ITCS únicamente del suelo del interior de todas las IF se apreció diferencias significativas entre las distintas IF (gl: 24; sig: 0.000). Lo mismo ocurrió para el ICTS del suelo de fuera de las IF (gl: 24; sig: 0.000). En un análisis más detallado el máximo del ICTS dentro del suelo de las IF fue de 0.665 y el mínimo de 0.401 , mientras que para fuera de la isla (suelo desnudo) los valores del ICTS fluctuaron entre 0.680 y 0.426 (Cuadro 3). En ambas condiciones se tienen sitios con moderada y buena calidad de acuerdo a la escala de Cantú (2007). Respecto a los subíndices de calidad física (SICF) y químico biológicas (SCQB) ambos presentaron un comportamiento muy similar tanto dentro como afuera, sin mostrar diferencias significativas. Los datos obtenidos del ICTS indican que hubo gran heterogeneidad al interior de cada grupo (dentro y fuera), pero pocas diferencias entre los suelos del interior y exterior con respecto a este índice. Estos resultados rompen con el supuesto que establecimos al suponer que el suelo del interior tendría una mejor calidad con respecto al de afuera. Sin embargo cabe la posibilidad de que la prueba utilizada para el análisis no fue lo suficientemente sensible para detectar las diferencias. 
El índice utilizado engloba muchas propiedades, dándole el mismo peso específico a cada una de ellas lo cual enmascara el efecto de propiedades que de acuerdo a varios autores (Aguilera et al., 1999; Xie y Steinberger, 2001; Gutiérrez y Squeo, 2004; Schade y Hobbie, 2005) son más determinantes en el funcionamiento ecológico de las IF. Tal y como lo plantean Schade y Hobbie (2005) y Bestelmeyer et al. (2006) las IF deben de estudiarse a una escala detallada enfocándose en aspectos que tienen que ver con la relación de patrones y procesos determinantes para su funcionamiento, como aquellos que tienen que ver con el reciclaje de nutrientes y el balance hídrico. Los últimos autores mencionan que para tener un entendimiento más completo del funcionamiento de estos ecosistemas no sólo se deben considerar los procesos que se dan a nivel superficial sino que también hay que analizar al suelo en su profundidad.

\section{Propiedades del Suelo}

No obstante que no se presentaron diferencias significativas en el índice de calidad total de los suelos tanto dentro como fuera de las IF, al analizar de manera individualizada las propiedades que se utilizaron para construir los subíndices e índice de calidad total con la prueba t-Student para muestras independientes, se observó que hubo propiedades como: densidad aparente, materia orgánica, $\mathrm{pH}$, magnesio y propiedades que tienen que ver con el agua en el suelo tales como: humedad en campo, humedad disponible y capacidad de campo, que mostraron diferencias significativas entre el suelo de dentro y el de afuera de las IF (Cuadro 4).

La materia orgánica fue una propiedad que mostró diferencia significativa, se concentró más al interior de las IF (2.30\%) (Cuadro 4) debido a los mecanismos de captura, donde los arbustos por medio del tallo, raíces salientes y el mantillo actúan como trampas colectoras de sedimentos y residuos orgánicos transportados por el agua y viento, formándose con el tiempo los montículos característicos de las IF y permitiendo asi la adquisiciòn de nutrimentos, materia orgánica y agua (Aber y Melillo, 2001). Los restos orgánicos que se acumulan bajo el dosel van enriqueciendo al suelo y proporcionando recursos para los habitantes de la IF (Gutiérrez y Squeo, 2004; Wilson y Thompson, 2005; Mora y Lázaro, 2014). Uno de los principales aportes de residuos orgánicos en las IF estudiadas es la producción de hojarasca aportada en este caso por el mezquite, al revisar la hojarasca acumulada dentro de las islas se pudo constatar que la mayor parte proviene del arbusto nodriza (mezquite), ya que los demás habitantes vegetales son diferentes especies de cactáceas cuya aportación orgánica es mínima. La composición química de la hojarasca de los arbustos nodriza es de vital importancia en el enriquecimiento del suelo (Wezel et al., 2000).

La densidad aparente es otra de las propiedades que mostró diferencia significativa, fue menor al interior de las IF generando con esto mayor porosidad, favoreciendo el desarrollo radicular y la infiltración (Holmgren y Scheffer, 2001). Al respecto Mora y Lázaro (2014) indican que abajo del dosel la densidad aparente disminuye debido a las entradas de materia orgánica y bioturbación lo que favorece la infiltración. Este comportamiento de la densidad aparente es el resultado de los mecanismos de realimentación sueloplanta responsable del patrón de distribución típico de la vegetación de las zonas áridas y semiáridas (Bochet et al., 1999; Maestre et al., 2005; Stavi et al., 2009). Shachak et al. (1998) mencionan que el suelo del exterior de las IF regularmente es más compacto y por lo tanto menos poroso, lo cual afecta a la disponibilidad de agua limitando así el establecimiento y crecimiento de plántulas (reclutamiento de nuevos individuos) dificultando con esto la recuperación del sistema. Por otra parte el suelo desnudo es más susceptible a la remoción de nutrientes por efecto de la erosión (Mora y Lázaro, 2014).

El pH y magnesio también presentaron diferencias significativas entre el suelo del interior de la isla y del exterior, no obstante que en ambas condiciones el $\mathrm{pH}$ fue moderadamente alcalino, el suelo del exterior obtuvo valores más elevados, algo similar ocurrió con el magnesio (Cuadro 4). Desafortunadamente hay poca información a la cuál recurrir en la literatura donde se mencione el comportamiento de estas propiedades en las IF, como para explicar al respecto; sin embargo, lo que si se reporta con frecuencia (Valiente-Banuet y Ezcurra, 1991; Holmgren y Scheffer, 2001; Gutiérrez y Squeo, 2004) es que el suelo abierto sin vegetación al estrés hídrico y salino se acentúa más por la exposición directa de los rayos solares incrementando con esto la evaporación y la concentración de sales. Por debajo del dosel de los arbustos las condiciones son menos estresantes hay una mayor infiltración y arrastre de sales 
Cuadro 4. Propiedades del suelo evaluadas dentro y fuera de las islas.

\begin{tabular}{|c|c|c|c|c|c|c|}
\hline \multirow[b]{2}{*}{ Propiedad } & \multicolumn{2}{|c|}{ Dentro } & \multicolumn{2}{|c|}{ Fuera } & \multicolumn{2}{|c|}{ Prueba t-Student } \\
\hline & $\begin{array}{l}\text { Promedio } \\
(25 \text { sitios })\end{array}$ & $\begin{array}{c}\text { Error } \\
\text { estándar }\end{array}$ & $\begin{array}{l}\text { Promedio } \\
(25 \text { sitios })\end{array}$ & $\begin{array}{c}\text { Error } \\
\text { estándar }\end{array}$ & $\mathrm{gl}$ & Sig. \\
\hline Arena $(\%)$ & 22.82 & 1.10 & 19.6 & 0.92 & 47.31 & 0.044 \\
\hline Limo $(\%)$ & 47.82 & 0.67 & 49.92 & 0.80 & 48.00 & 0.050 \\
\hline Arcilla $(\%)$ & 29.37 & 0.75 & 30.21 & 0.82 & 48.00 & 0.466 \\
\hline Densidad aparente $\mathrm{kg} \mathrm{m}^{-3}$ & 1.10 & 0.02 & 1.14 & 0.02 & 48.00 & $0.040^{*}$ \\
\hline Porosidad \% & 55.47 & 1.16 & 53.85 & 0.71 & 48.00 & 0.240 \\
\hline Relación agua/aire & 0.67 & 0.02 & 0.66 & 0.01 & 48.00 & 0.371 \\
\hline Conductividad eléctrica $\mathrm{dS} \mathrm{m}^{-1}$ & 1.57 & 0.46 & 1.61 & 0.84 & 48.00 & 0.973 \\
\hline Salinidad \% & 0.92 & 0.27 & 0.99 & 0.49 & 48.00 & 0.904 \\
\hline Materia orgánica \% & 2.30 & 0.17 & 1.41 & 0.11 & 48.00 & $0.000^{*}$ \\
\hline $\mathrm{pH}$ (agua) rel 1:2.5 & 7.68 & 0.05 & 7.89 & 0.04 & 48.00 & $0.003^{*}$ \\
\hline CICT cmol kg-1 & 27.42 & 0.62 & 27.18 & 0.52 & 48.00 & 0.770 \\
\hline Calcio cmol kg-1 & 11.48 & 0.27 & 11.54 & 0.33 & 48.00 & 0.870 \\
\hline Magnesio $\mathrm{cmol} \mathrm{kg}^{-1}$ & 2.09 & 0.22 & 2.85 & 0.24 & 48.00 & $0.022 *$ \\
\hline Sodio cmol kg-1 & 4.91 & 0.54 & 5.68 & 0.45 & 48.00 & 0.287 \\
\hline Potasio $\mathrm{cmol} \mathrm{kg}^{-1}$ & 3.76 & 0.27 & 3.12 & 0.25 & 48.00 & 0.091 \\
\hline Saturación de bases \% & 81.16 & 1.42 & 85.47 & 1.08 & 48.00 & $0.027 *$ \\
\hline Nitrógeno total \% & 0.18 & 0.02 & 0.12 & 0.01 & 48.00 & $0.000 *$ \\
\hline Fósforo asimilable ppm & 1.01 & 0.10 & 1.15 & 0.09 & 48.00 & 0.293 \\
\hline Humedad en campo & 45.94 & 0.87 & 42.01 & 1.08 & 48.00 & $0.007 *$ \\
\hline Capacidad de campo \% & 37.01 & 0.49 & 35.22 & 0.46 & 48.00 & $0.010^{*}$ \\
\hline Humedad aprovechable \% & 17.37 & 0.22 & 16.54 & 0.22 & 48.00 & $0.009 *$ \\
\hline Capacidad de aireación \% & 14.87 & 1.44 & 13.58 & 1.17 & 48.00 & 0.143 \\
\hline Temperatura del suelo ${ }^{\circ} \mathrm{C}$ & 32.28 & 0.46 & 39.32 & 1.03 & 48.00 & $0.000 *$ \\
\hline
\end{tabular}

* Significancia $\alpha<0.05$.

al subsuelo (Titus et al., 2002; Gutiérrez y Squeo, 2004).

Todas las variables evaluadas relacionadas con la presencia del agua en el suelo (humedad en campo, capacidad de campo y humedad disponible) mostraron diferencias significativas entre el suelo del interior y exterior de las IF, manifestando con claridad el efecto que tiene la IF sobre la captación y retención de agua. Los suelos del interior captan y retienen más agua, lo cual se refleja en porcentaje de agua capturada y agua disponible (Cuadro 4). Estos resultados son acordes a los encontrados por Pate y Dawson (1999), Gutiérrez y Squeo (2004), Ridolfi et al. (2008), en el sentido de que una de las funciones ecológicas más importantes que desarrollan las IF es la reducción del estrés hídrico. La hojarasca depositada bajo el arbusto nodriza permite que la precipitación sea absorbida por las capas superiores del suelo y quede disponible para las plantas (Shachak et al., 1998). Aunado a esto León y Squeo (2004) mencionan que los arbustos con raíces pivotantes como el mezquite alcanzan capas freáticas profundas efectuando un levantamiento hidráulico del agua subterránea, lo que conduce a una disminución del estrés hídrico este levantamiento hidráulico contribuye a mantener húmeda la superficie del suelo facilitando así la solubilización de nutrientes y promoviendo los procesos microbianos (Dawson, 1993).

\section{CONCLUSIÓN}

No se encontraron diferencias significativas en el índice de calidad total del suelo, ni en los subíndices de calidad física y químico biológica utilizados para evaluar la calidad del suelo tanto del interior como exterior de las islas de fertilidad, sin embargo hubo diferencias en 
densidad aparente, materia orgánica, $\mathrm{pH}$, magnesio, humedad en campo, humedad disponible y capacidad de campo. Estos resultados indican o sugieren que este tipo de patrón de distribución de islas de fertilidad no puede ser estudiado de una manera general o global a través de índices los cuales involucran a conjuntos de variables a las cuales se les da el mismo peso específico, sino que debe ponderarse la importancia que tiene cada propiedad en el funcionamiento de las islas de fertilidad (materia orgánica, la actividad microbiológica, el reciclaje de nutrientes y todo lo que tiene que ver con la relación suelo-plantaagua). Se pudo comprobar que uno de los principales agentes responsables en la variación espacial de las propiedades del suelo en el ecosistema estudiado es la propia vegetación, en este sentido la influencia que tiene la planta nodriza es determinante en la regulación y variación de propiedades que resultan determinantes para el funcionamiento de estos ecosistemas. Las nodrizas a través de una serie de mecanismos de realimentación permiten la construcción de un micro nicho el cual logra permanecer por largos periodos.

\section{LITERATURA CITADA}

Aber, J. D. and J. M. Melillo. 2001. Terrestrial ecosystems. Brooks Cole. Pacific Grove, CA, USA.

Aguilera, L. E., J. R. Gutiérrez, and P. L. Meserve. 1999. Variation in soil micro-organisms and nutrients underneath and outside the canopy of Adesmia bedwellii (Papilionaceae) shrubs in arid coastal Chile following drought and above average rainfall. J. Arid Environ. 42: 61-70.

Andrews, S. S., D. L. Karlen, and J. P. Mitchel. 2002. A comparison of soil quality indexing methods for vegetable production systems in Northern California. Agric. Ecosyst. Environ. 90: $25-45$.

Berg, N. and Y. Steinberger. 2012. The role of perennial plants in preserving annual plant complexity in a desert ecosystem. Geoderma 185-186: 6-11.

Bochet, E., J. L. Rubio, and J. Poesen. 1999. Modified topsoil islands within patchy Mediterranean vegetation in SE Spain. Catena 38: 23-44.

Bonanomi, G., M. Rietkerk, S. C. Dekker, and S. Mazzoleni. 2007. Islands of fertility induce co-occurring negative and positive plant-soil feedbacks promoting coexistence. Plant Ecol. 197: 207-218.

Bestelmeyer, B. T., J. P. Ward, J. E. Herrick, and A. J. Tugel. 2006. Fragmentation effects on soil aggregate stability in a patchy arid grassland. Rangeland Ecol. Manage. 59: 406-415.

Cantú, M. P., A. Becker, J. C. Bedano y H. F. Schiavo. 2007. Evaluación de la calidad de suelos mediante el uso de indicadores e índices. Cienc. Suelo (Argentina) 25: 173-178.

Dawson, T. E. 1993. Hydraulic lift and water use by plants: Implications for water balance, performance and plant-plant interactions. Oecologia 95: 565-574.
Flores, J. and E. Jurado. 2003. Are nurse-protégé interactions more common among plants from arid environments? J. Veget. Sci. 14: 911-916.

García, E. 2004. Modificaciones al sistema de clasificación climática de Köppen: Para adaptarlo a las condiciones de la República Mexicana. Instituto de Geografía UNAM. México, D. F.

Gugino, B. K., O. J. Idowu, R. R. Schindelbeck, H. M. van Es, D. W. Wolfe, B. N. Moebius-Clune, J. E. Thies, and G. S. Abawi. 2009. Cornell soil health assessment training manual. Cornell University College of Agriculture and Life Sciences. Cornell Digital Print Services. Ithaca, NY, USA.

Gutiérrez, J. R. y F. A. Squeo. 2004. Importancia de los arbustos leñosos en los ecosistemas semiáridos de Chile. Ecosistemas 13: 36-45.

Herrick, J. E., J. R. Brown, A. J. Tugel, P. L. Shaver, and K. M. Havstad. 2002. Application of soil quality to monitoring and management: Paradigms from rangeland ecology. Agron. J. 94: 3-11.

Holmgren, M. and M. Scheffer. 2001. El Niño as a window of opportunity for the restoration of degraded arid ecosystems. Ecosystems 4: 151-159.

León, M. F. y F. A. Squeo. 2004. Levantamiento hidráulico: La raíz del asunto. pp. 99-100. In: H. M. Cabrera (ed.). Fisiología ecológica en plantas: Mecanismos y respuestas a estrés en los ecosistemas. Departamento de Biología, Facultad de Ciencias, Universidad de La Serena. Valparaíso, Chile.

McAuliffe, J. R. 1988. Markovian dynamics of simple and complex desert plant communities. Am. Nat. 131: 459-490.

Maestre, F.T., A. Escudero, I. Martinez, C. Guerrero, and A. Rubio, 2005. Does spatial pattern matter to ecosystem functioning? Insights from biological soil crusts. Funct. Ecol. 19: 566-573.

Mora, J. L. and R. Lázaro. 2014. Seasonal changes in bulk density under semiarid patchy vegetation: The soil beats. Geoderma 235-236: 30-38.

Muñoz I., D. J., F. G. López., M. M. Hernández, A. A. Soler y G. J. López. 2009. Impacto de la pérdida de la vegetación en las propiedades de un suelo aluvial. Terra Latinoamericana 27: 237-246.

Pate, J. S. and T. E. Dawson. 1999. Assessing the performance of woody plants in uptake and utilization of carbon, water and nutrients. Implications for designing agricultural mimic systems. Agrofor. Syst 45: 245-275.

Peters, P. C. D., B. T. Bestelmeyer, E. J. Herrick, E. L. Fredrickson, H. C. Monger, and K. M. Havstad. 2006. Disentangling complex landscapes: New insights into arid and semiarid system dynamics. BioScience 56: 491- 501.

Ridolfi, L., F. Laio, and P. D'Odorico. 2008. Fertility island formation and evolution in dryland ecosystems. Ecol. Soc. 13: 5 .

Reynolds, W. D., B. T. Bowman, C. F. Drury, C. S. Tan, and X. Lu. 2002. Indicators of good soil physical quality: Density and storage parameters. Geoderma 110: 131-146.

Shepherd, T. G. 2000. Visual soil assessment. Volume 1. Field guide for cropping and pastoral grazing on flat to rolling country. Horizons.mw / Landcare Research. Palmerston North, New Zealand.

Schade J. D. and S. E. Hobbie. 2005. Spatial and temporal variation in islands of fertility in the Sonora Desert. Biogeochemistry 73: 541-553. 
Shachak, M., M. Sachs, and I. Moshe. 1998. Ecosystem management of desertified shrublands in Israel. Ecosystems 1: 475- 483.

Silva, A., J. Ponce de León, F. García y A. Durán. 1988. Aspectos metodológicos en la determinación de la capacidad de retener agua de los suelos del Uruguay. Boletín de Investigación $\mathrm{N}^{\mathrm{o}}$ 10. Facultad de Agronomía. Universidad de la República. Uruguay.

Skopp, J., M. D. Jawson, and J. W Doran. 1990. Steady-state aerobic microbial activity as a function of soil water content. Soil Sci. Soc. Am. J. 54: 1619-1625.

Stavi, I., H. Lavee, E.D. Ungar, and P. Sarah. 2009. Ecogeomorphic feedbacks in semiarid rangelands: A review. Pedosphere 19: 217-229.

Tewksbury, J. J. and J. D. Lloyd. 2001. Positive interactions under nurse-plants: Spatial scale, stress gradients and benefactor size. Oecologia 127: 425-434.

Tielborger, K. and R. Kadmon. 2000. Indirect effects in a desert plant community: Is competition among annuals more intense under shrub canopies? Plant Ecol. 150: 53-63.

Titus, J. H., R. S. Nowak, and S. D. Smith. 2002. Soil resource heterogeneity in the Mojave Desert. Arid Environ. 52: 269292.

Turner, R. M. and D. E. Brown. 1994. Tropical-subtropical desertlands: Sonoran desertscrub. pp. 154.1 In: D. E. Brown (ed.). Biotic communities southwestern United States and Northwestern Mexico. University of Utah Press. Salt Lake. UT, USA.
UBIPRO (Unidad de Biología y Prototipos). 2007. Deterioro ambiental en zonas áridas. Facultad de Estudios Superiores Iztacala, UNAM. México, D. F.

Valiente-Banuet, A. and E. Ezcurra. 1991. Shade as a cause of the association between the cactus Neobuxxaunia teterzo and the nurse plant Mimosa luisana. J. Ecol. 79: 961-971.

Wezel, A., J. L. Rajot, and C. Herbrig. 2000. Influence of shrubs on soil characteristics and their function in Sahelian agroecosystems in semi-arid Niger. J. Arid Environ. 44: 383-398.

Wilson, T. B. and T. L. Thompson. 2005. Soil nutrient distributions of mesquite-dominated desert grasslands: Changes in time and space. Geoderma 126: 301-315.

Whitford, W. G. 2002. Ecology of desert systems. Academic Press. London, UK.

Westoby, M., B. Walker, and I. Noy-Meir. 1989. Opportunistic management for rangelands not at equilibrium. J. Range Manage. 42: 266-274.

Xie, G. and Y. Steinberger. 2001. Temporal patterns of C and N under shrub canopy in a loessial soil desert ecosystem. Soil Biol. Biochem. 33: 1371-1379.

USDA (United States Department of Agriculture). 1999. Soil Quality Test Kit Guide. Agricultural Research Services, Natural Resources Conservation Service, Soil Quality Institute. Washington, DC, USA.

Zar, J. 1999. Biostatistical analysis. Prentince Hall. Upper Saddle River, NJ, USA. 